\title{
Principal Component Analysis of Phenolic Acid Spectra
}

\author{
Ronald A. Holser \\ Richard Russell Research Center, USDA-ARS, 950 College Station Road, Athens, GA 30605, USA \\ Correspondence should be addressed to Ronald A. Holser, ronald.holser@ars.usda.gov
}

Received 12 September 2012; Accepted 3 October 2012

Academic Editors: A. Tadjeddine and H. Yang

Copyright () 2012 Ronald A. Holser. This is an open access article distributed under the Creative Commons Attribution License, which permits unrestricted use, distribution, and reproduction in any medium, provided the original work is properly cited.

\begin{abstract}
Phenolic acids are common plant metabolites that exhibit bioactive properties and have applications in functional food and animal feed formulations. The ultraviolet (UV) and infrared (IR) spectra of four closely related phenolic acid structures were evaluated by principal component analysis (PCA) to develop spectral models for their rapid detection. Results demonstrated that UV and IR spectra could discriminate between each of the phenolic acids in overall models. Calculation of model scores and loadings showed that derivative UV spectra accounted for $99 \%$ variation with 2 principal components (PC) while derivative IR spectra required 3 PCs. Individual PCA models were developed for ferulic acid and $p$-coumaric acid using derivative UV spectra for detection and classification by soft independent modeling of class analogy (SIMCA). The application of this spectral technique as a classification model is expected to promote the use of agricultural residues as a source of these phenolic compounds.
\end{abstract}

\section{Introduction}

Low molecular weight phenolic compounds such as ferulic acid and coumaric acid exhibit antioxidant and antimicrobial properties that add health benefits to foods and animal feeds $[1,2]$. The bioactivities of these compounds provide functionality and extend the shelf life of formulated products. Current sources of phenolic acids include the commodity cereal grains, for example, corn, oat, and wheat. Due to the recent cost increase of these materials alternative sources such as lignocellulosic biomass offers promise as bioethanol industry develops. Switchgrass and other renewable energy crops contain significant amounts of phenolic acids that could be recovered as coproducts and improve bioethanol production $[3,4]$.

The development of a spectroscopic technique to rapidly detect and characterize the phenolic acid content profile could replace the more time consuming standard chromatographic analysis. Analysis times between 30 and 60 minutes are typically needed for separation of phenolic compounds by liquid chromatography. Quantitative results require replicate analysis of each sample and technical expertise to prepare samples and operate the chromatograph reproducibly. In contrast, a minimal amount of sample preparation is needed for spectroscopic methods and the instrumentation is less expensive to purchase and maintain. A modern instrument can scan a sample in less than 2 minutes and is routinely performed by operators with a small amount of training.

The chemical structure of phenolic compounds, specifically the aromatic ring, produces strong absorbance in the ultraviolet (UV) region associated with electronic transitions of the molecule. This provides a unique spectrum in addition to absorbance in the infrared (IR) region due to vibrational molecular motions. Spectra were collected over these regions for structurally related compounds (Figure 1) and evaluated using principal component analysis (PCA) to develop a rapid detection method $[5,6]$.

\section{Experimental}

2.1. Spectroscopy. Infrared spectra were collected on a Perkin Elmer model Spectrum 400 FTIR spectrometer equipped with a diamond crystal ATR (Waltham, MA, USA). Standards were prepared from $98 \%$ pure ferulic acid, $p$-coumaric acid, 3,5-dimethoxy-4-methoxy cinnamic acid, and 3,4dihydroxy cinnamic acid (Sigma-Aldrich, St. Louis, MO, USA) dissolved in anhydrous ethanol (AAper, Shelbyville, $\mathrm{KY}$ ). A few drops were placed on the ATR and scanned from 650 to $4000 \mathrm{~cm}^{-1}$ with a resolution of $4 \mathrm{~cm}^{-1}$. Each recorded 
<smiles>O=C(O)/C=C/c1ccc(O)cc1</smiles>

p-coumaric acid<smiles>O=C(O)/C=C/c1ccc(O)c(O)c1</smiles>

3,4-dihydroxycinnamic acid<smiles>COc1cc(/C=C/C(=O)O)ccc1O</smiles>

Ferulic acid<smiles>COc1cc(/C=C/C(=O)O)cc(OC)c1O</smiles>

3,5-dimethoxy-4-hydroxycinnamic acid

FIgure 1: Phenolic acid structures investigated by UV and IR spectroscopy.

spectrum was the result of 32 coadded scans. Ultraviolet spectra were collected on a Perkin Elmer model Lambda $2 \mathrm{~S}$ UV/VIS spectrometer (Waltham, MA, USA). Phenolic acid standards were diluted to 1,5 , and $10 \mu \mathrm{g} / \mathrm{mL}$ and scanned in $3.5 \mathrm{~mL}$ quartz cuvettes. UV spectra were collected from 200 to $400 \mathrm{~nm}$ at $240 \mathrm{~nm} / \mathrm{min}$ with a $2 \mathrm{~nm}$ slit width. A minimum of five replicates were collected.

2.2. Data Analysis. Spectra were analyzed using the Unscrambler X (Camo Software, Oslo, Norway). Derivative spectra were calculated by the Savitsky-Golay method. Principal component analysis (PCA) was performed on smoothed and first derivative spectra to determine potential grouping for subsequent classification. Classes were established from the spectra of each phenolic acid standard for Soft Independent Modeling of Class Analogy (SIMCA) using individual PCA models [7].

\section{Results and Discussion}

The UV spectra of ferulic acid and p-coumaric acid show clear differences over the scan range of $200-400 \mathrm{~nm}$ (Figure 2). Ferulic acid exhibits a maximum absorbance at

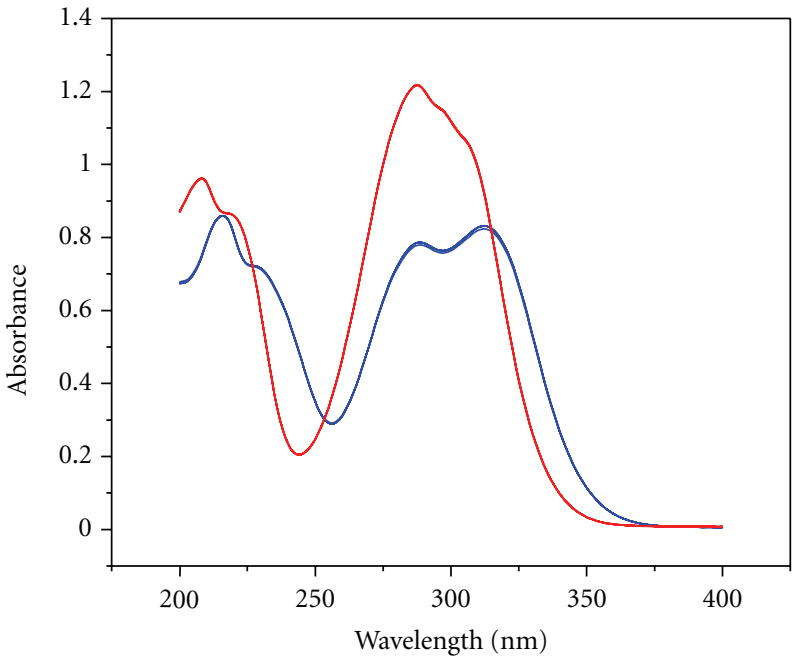

Figure 2: UV spectra of ferulic acid (blue) and p-coumaric acid (red).

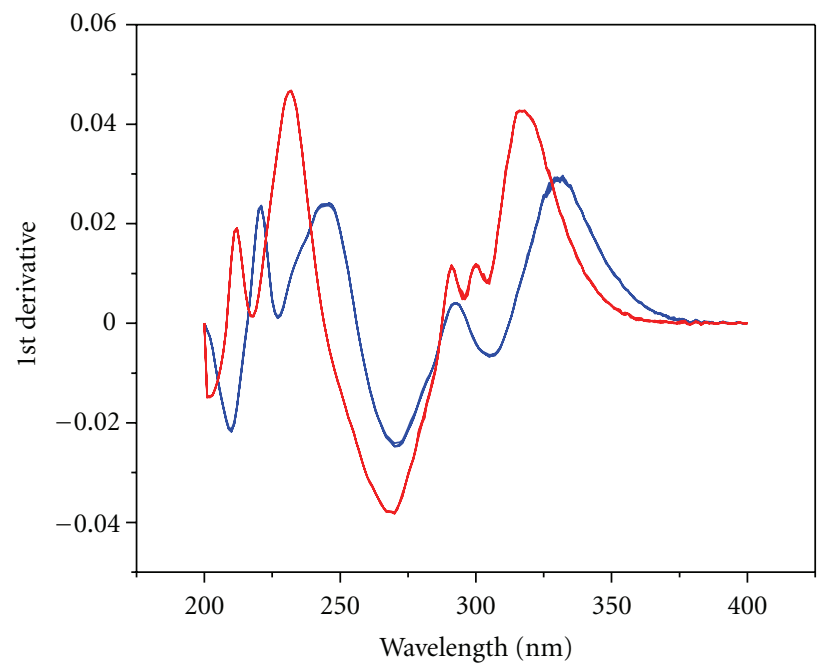

FIGURE 3: First derivative UV spectra of ferulic acid (blue) and $p$ coumaric acid (red).

$215 \mathrm{~nm}$ with additional absorbances at $287 \mathrm{~nm}$ and $312 \mathrm{~nm}$. In contrast, $p$-coumaric acid displays a maximum absorbance at $286 \mathrm{~nm}$ with additional absorbances at $209 \mathrm{~nm}$ and $220 \mathrm{~nm}$. Applying the first derivative transform to these spectra produced the results shown in Figure 3. This provided better resolution between the two phenolic acids without introducing significant noise. Maxima occur in the derivative spectra at $231 \mathrm{~nm}$ and $315 \mathrm{~nm}$ for $p$-coumaric acid and $220 \mathrm{~nm}, 242 \mathrm{~nm}$, and $328 \mathrm{~nm}$ for ferulic acid. The corresponding infrared spectra are shown in Figures 4 and 5. The appearance is more complicated than the UV spectra but differences between ferulic acid and p-coumaric acid are evident in the $650-1500 \mathrm{~cm}^{-1}$ region. The first derivative IR spectra display sharper absorbances and a smoother baseline.

Spectra were initially evaluated by principal component analysis (PCA) to determine if sufficient differences were 


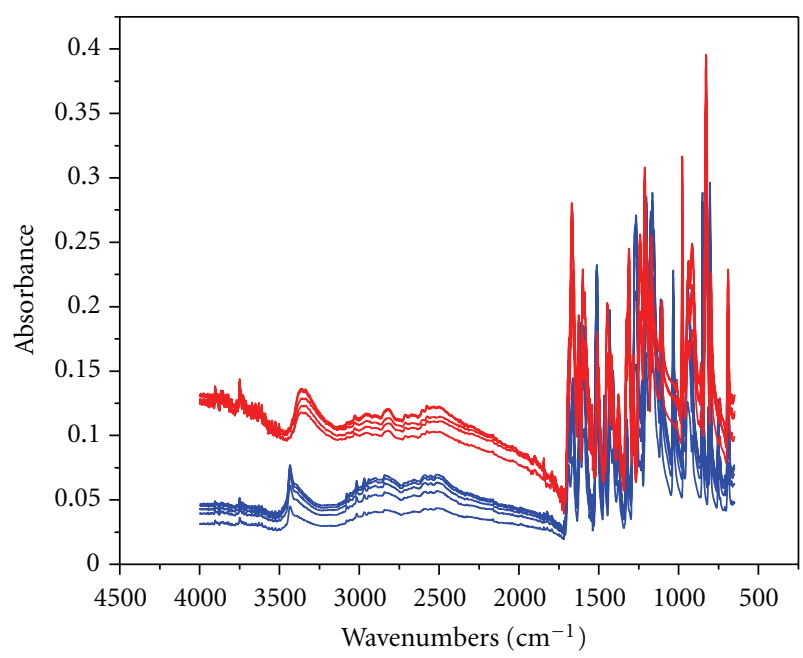

Figure 4: IR spectra of ferulic acid (blue) and p-coumaric acid (red).

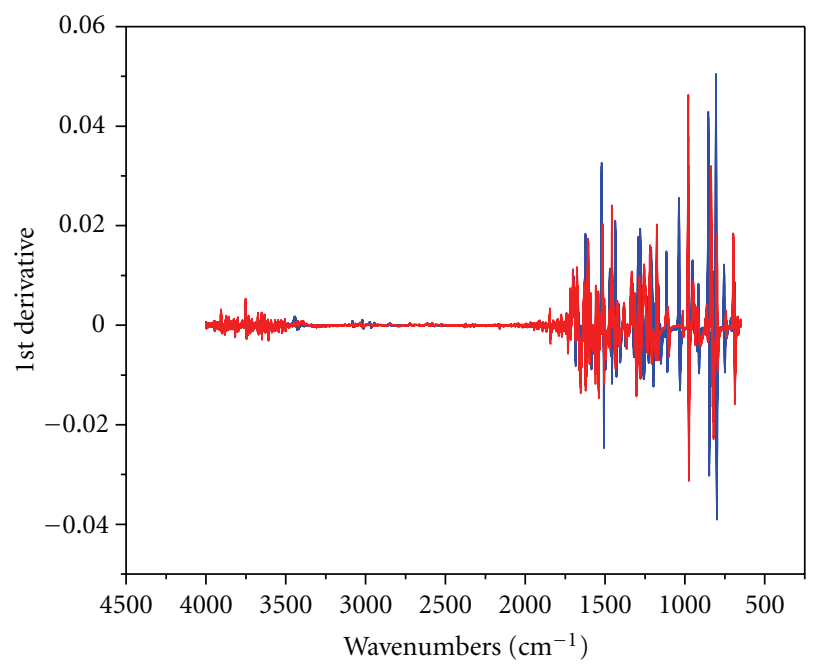

FIGURE 5: First derivative IR spectra of ferulic acid (blue) and $p$ coumaric acid (red).

evident to form the basis of a classification scheme. PCA was implemented using the NIPALS algorithm with cross validation and mean centered data. The scores and loadings plots were examined for grouping of coumaric acid and ferulic acid. The analysis of UV spectra produced score plots showing the two acids clearly separated along the $x$-axis with coumaric acid at 1.4 and ferulic acid at -1.4 . The corresponding loadings plot is shown in Figure 6. The maximum loading value occurred near the center $(285 \mathrm{~nm})$ of a broad peak between $325 \mathrm{~nm}$ and $240 \mathrm{~nm}$. For comparison, the first derivative spectra analyzed with the same parameters provided similar grouping of $p$-coumaric acid and ferulic acid with separation along the $x$-axis. The loadings plot is shown in Figure 7 where three spectral regions with maxima at 211,229 , and $314 \mathrm{~nm}$ are observed. This preliminary analysis of UV spectra showed good separation of the two acids into groups described by a single principal component.

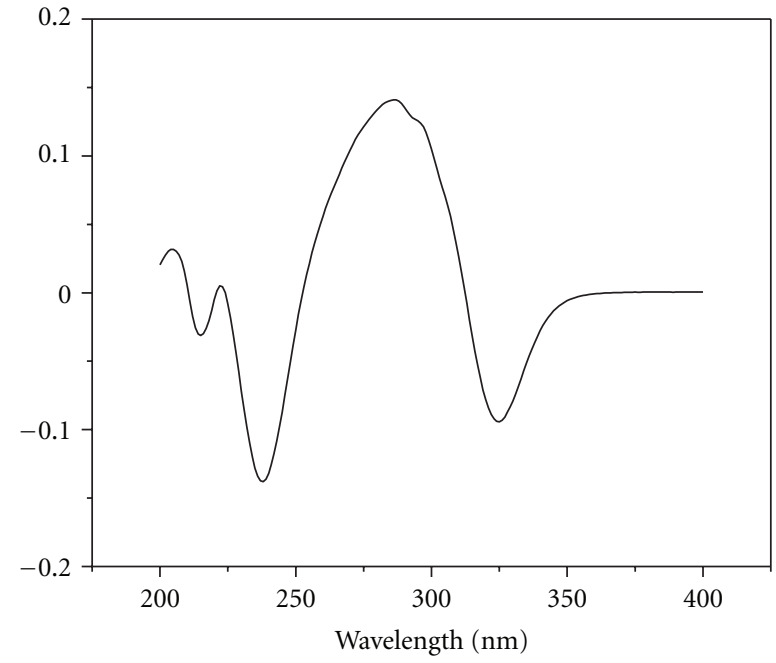

FIGURE 6: Loadings plot for UV spectra PCA model.

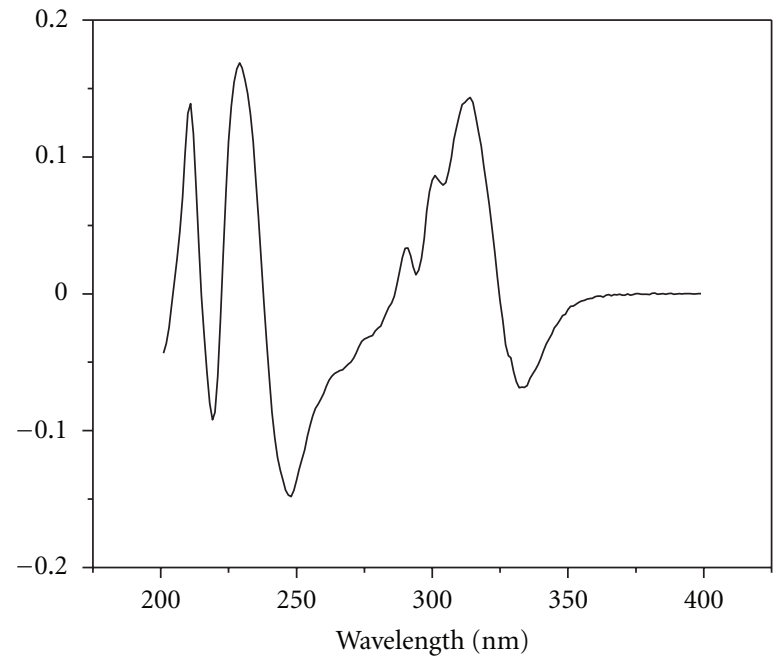

FIGURE 7: Loadings plot for first derivative UV spectra PCA model.

IR spectra were evaluated by PCA also using the NIPALS algorithm with mean centered data. Analysis of the scores plot showed $92 \%$ of the variation was accounted by the first principal component (PC) and 7\% by the second PC. Analysis of the derivative spectra showed $97 \%$ of the variation accounted for by the first PC and 3\% by the second PC. Scores and loadings plots are displayed in Figures 8 and 9 for the IR spectra and Figures 10 and 11 for the derivative IR spectra.

The preliminary PCA results obtained with UV and IR spectra demonstrated the ability of models based on both spectral regions to clearly distinguish $p$-coumaric acid from ferulic acid. This technique was further tested by introducing two additional phenolic compounds of similar structure. Ferulic acid (4-hydroxy-3-methoxycinnamic acid) and $p$-coumaric acid (4-hydroxycinnamic acid) are presented in Figure 1 with the related compounds 3,5-dimethoxy4-hydroxycinnamic acid and 3,4-dihydroxycinnamic acid. 


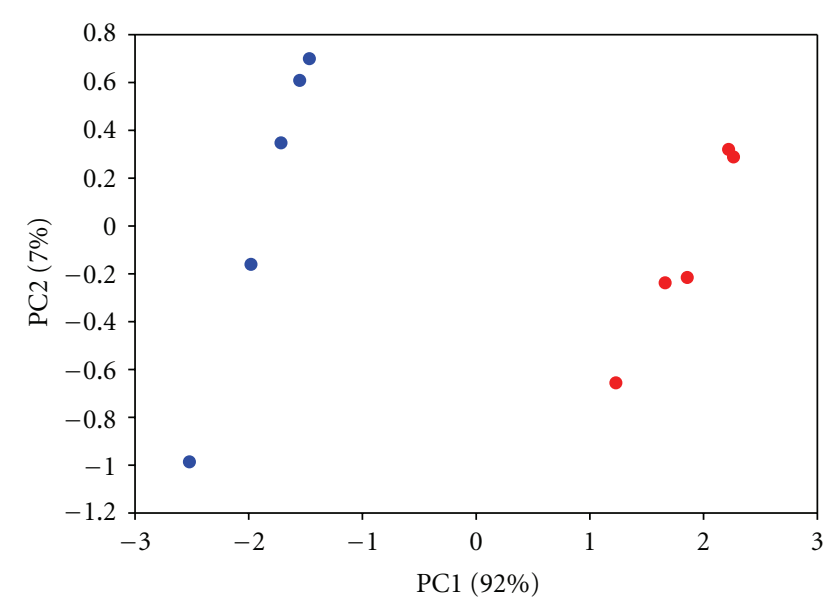

FIGURE 8: IR spectra scores plot for PCA model of ferulic acid (blue) and $p$-coumaric acid (red).

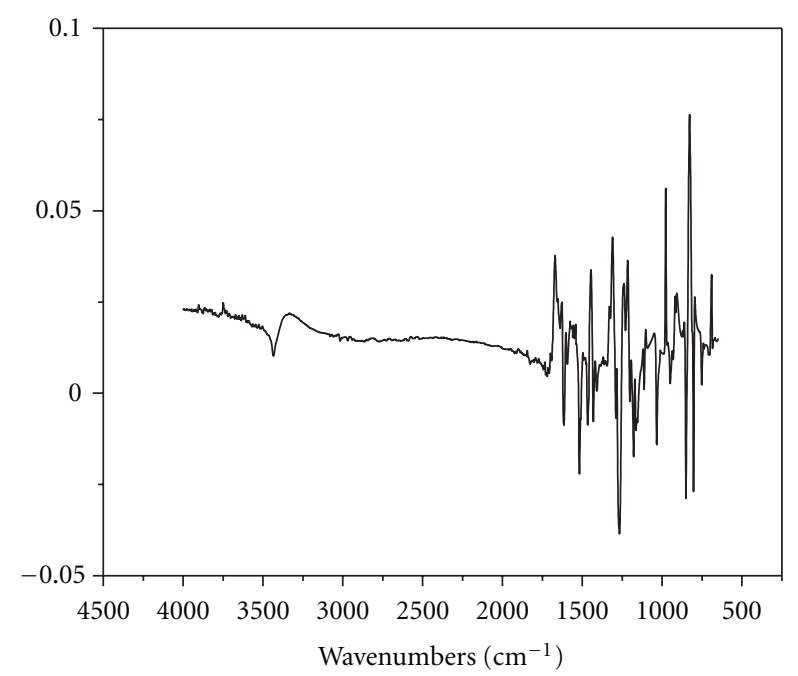

FIGURE 9: IR spectra loadings plot for $p$-coumaric acid/ferulic acid PCA model.

The differences between these four compounds are slight and involve the number and location of the substituent hydroxy and methoxy groups on the ring. UV and IR spectra of these phenolic compounds were evaluated as before with PCA to determine the ability to discriminate between such similar structures.

PCA results for the derivative spectra are presented in Figures 12 and 13 for models based on IR and UV, respectively. Derivative IR spectra accounted for 99\% variation with 3 principal components and showed separation of all four phenolic compounds. The derivative UV spectra accounted for $99 \%$ of the variation with 2 principal components. Based on these results the derivative UV spectra were selected for the classification of phenolic compounds.

\section{Conclusion}

The application of UV spectroscopy with principal component analysis provided an effective method to classify

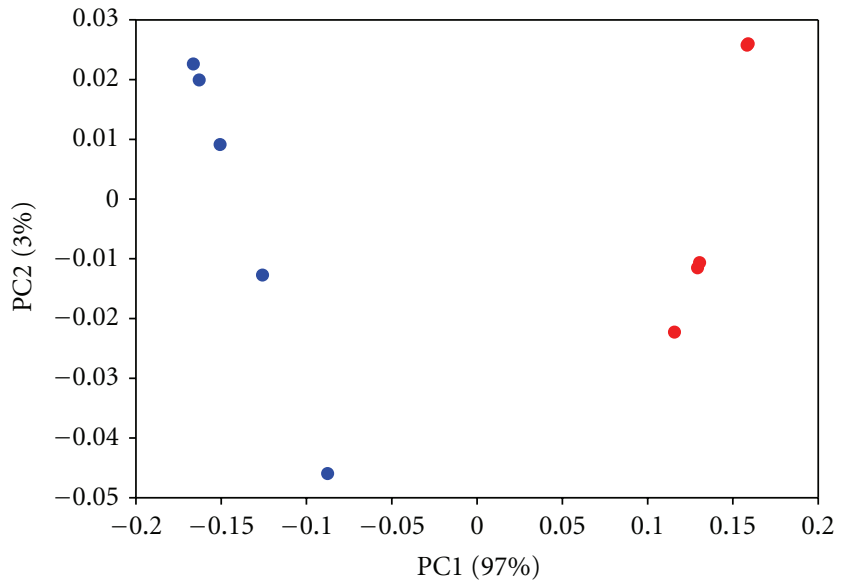

FIgURe 10: First derivative IR spectra scores plot for PCA model of ferulic acid (blue) and $p$-coumaric acid (red).

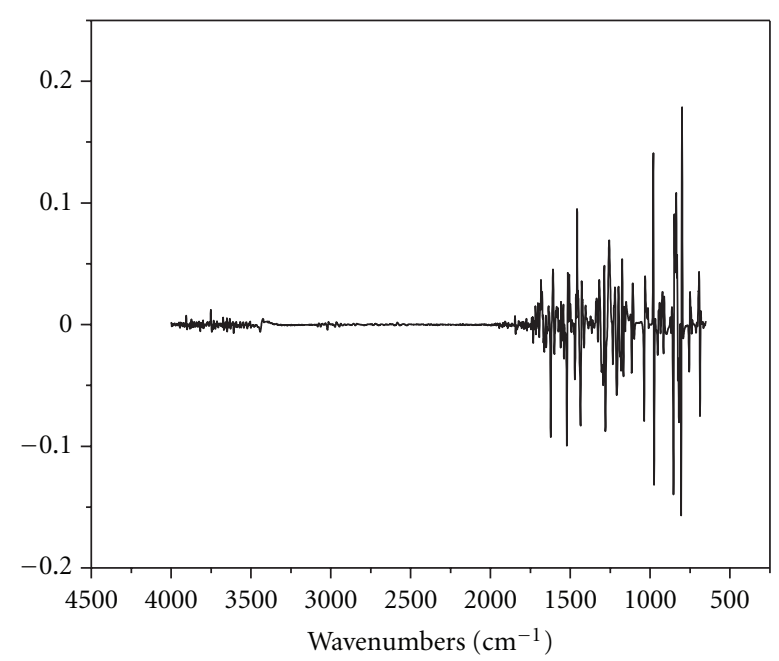

FIGURE 11: First derivative IR spectra loadings plot for $p$-coumaric and ferulic acids PCA model.

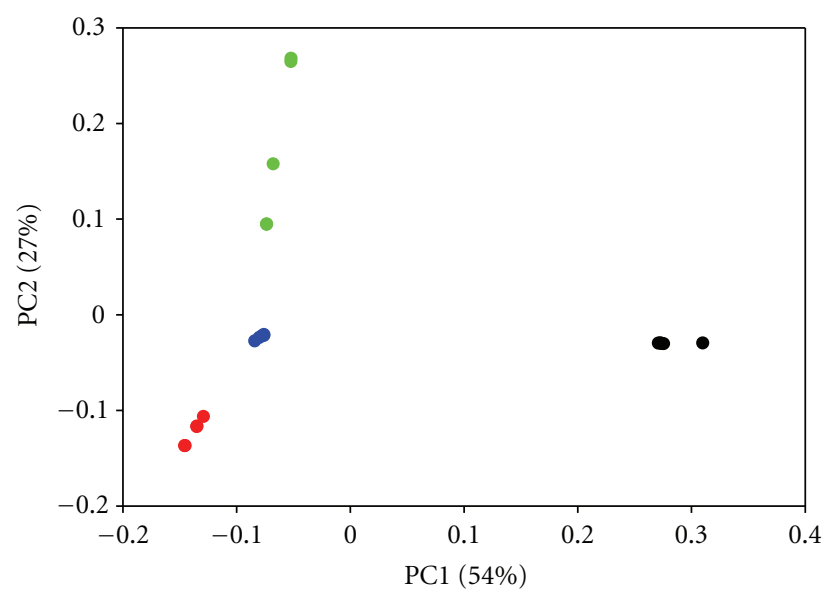

FIGURE 12: Scores plot for first derivative IR spectra of $p$-coumaric acid (red); ferulic acid (blue); 3,4-dihydroxycinnamic acid (green); 3,5-dimethoxy-4-hydroxycinnamic acid (black). 


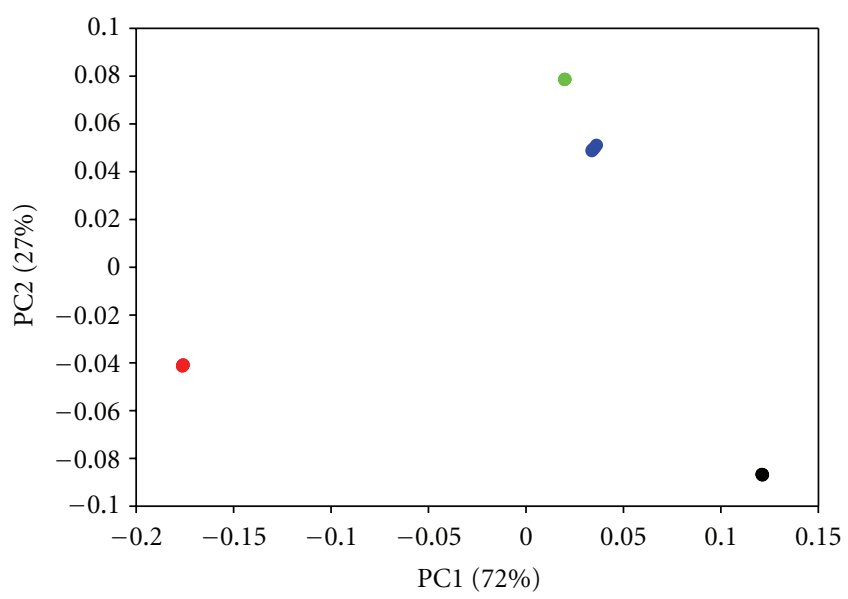

FIGURE 13: Scores plot for first derivative UV spectra of $p$-coumaric acid (red); ferulic acid (blue); 3,4-dihydroxycinnamic acid (green); 3,5-dimethoxy-4-hydroxycinnamic acid (black).

p-coumaric acid and ferulic acid based on spectral characteristics. This approach offers a rapid nondestructive technique to detect and classify phenolic compounds that can be extended to include structurally similar compounds. The application of these results will be made to identify such bioactive natural products in agricultural materials for use in foods and animal feeds.

\section{References}

[1] B. Baurhoo, C. A. Ruiz-Feria, and X. Zhao, "Purified lignin: nutritional and health impacts on farm animals-a review," Animal Feed Science and Technology, vol. 144, no. 3-4, pp. 175184, 2008.

[2] K. J. Jenkins, F. W. Collins, and M. Hidiroglou, "Research note: efficacy of various flavonoids and simple phenolics in prevention of nutritional myopathy in the chick," Poultry science, vol. 71, no. 9, pp. 1577-1580, 1992.

[3] A. Berchieri and P. A. Barrow, "The antibacterial effects for Salmonella Enteritidis phage type 4 of different chemical disinfectants and cleaning agents tested under different conditions," Avian Pathology, vol. 25, no. 4, pp. 663-673, 1996.

[4] D. Zhang, H. Hu, Q. Rao, and Z. Zhao, "Synergistic effects and physiological responses of selected bacterial isolates from animal feed to four natural antimicrobials and two antibiotics," Foodborne Pathogens and Disease, vol. 8, no. 10, pp. 1055-1062, 2011.

[5] H. Hotelling, "Analysis of a complex of statistical variables into principal components," Journal of Educational Psychology, vol. 24, no. 6, pp. 417-441, 1933.

[6] R. A. Darton, "Rotation in factor analysis," The Statistician, vol. 29, no. 3, pp. 167-194, 1980 .

[7] M. Insausti, A. A. Gomes, F. V. Cruz et al., "Screening analysis of biodiesel feedstock using UV-vis, NIR and synchronous fluorescence spectrometries and the successive projections algorithm," Talanta, vol. 97, pp. 579-583, 2012. 


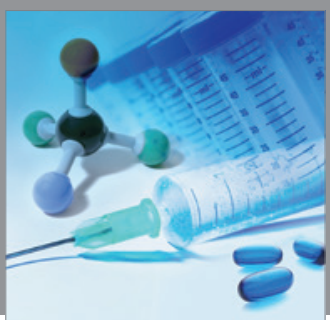

International Journal of

Medicinal Chemistry

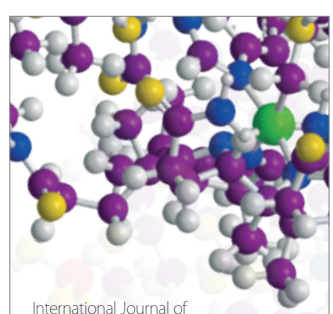

Carbohydrate Chemistry

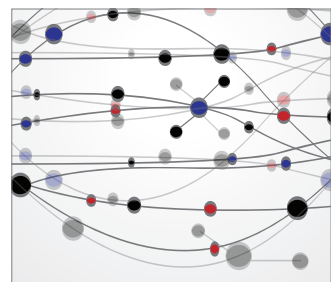

The Scientific World Journal
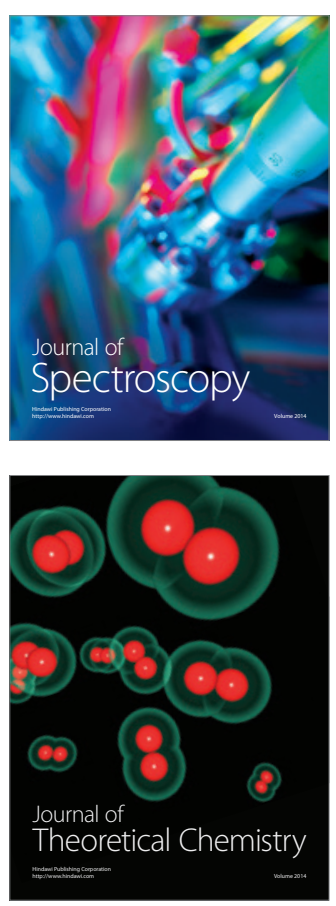
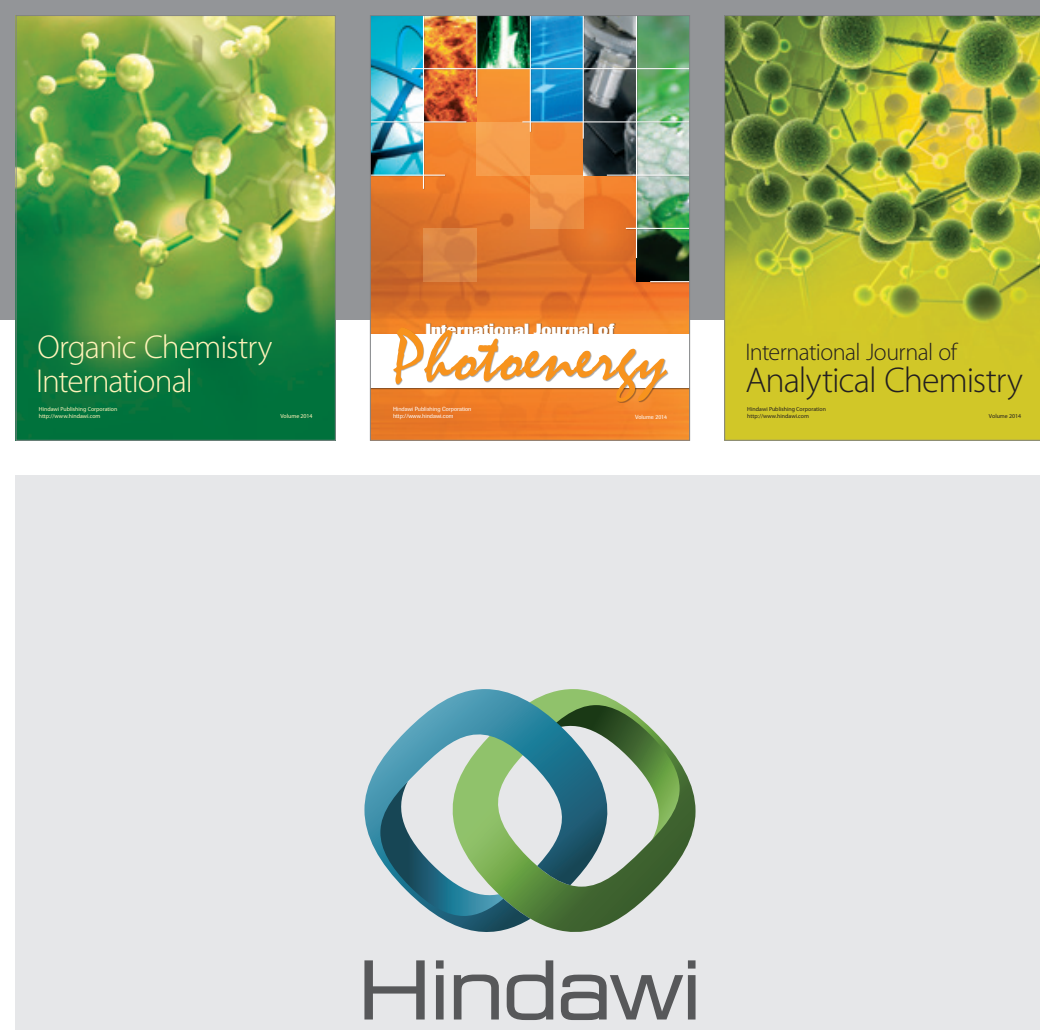

Submit your manuscripts at

http://www.hindawi.com
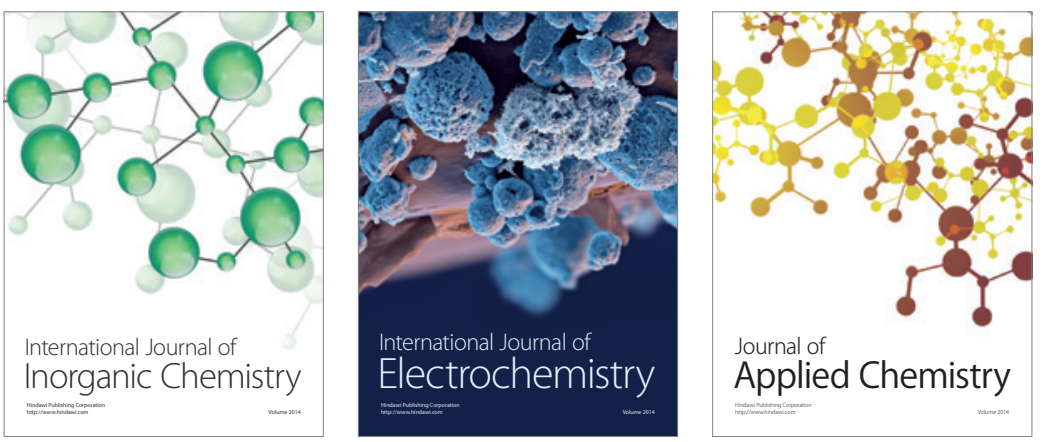

Journal of

Applied Chemistry
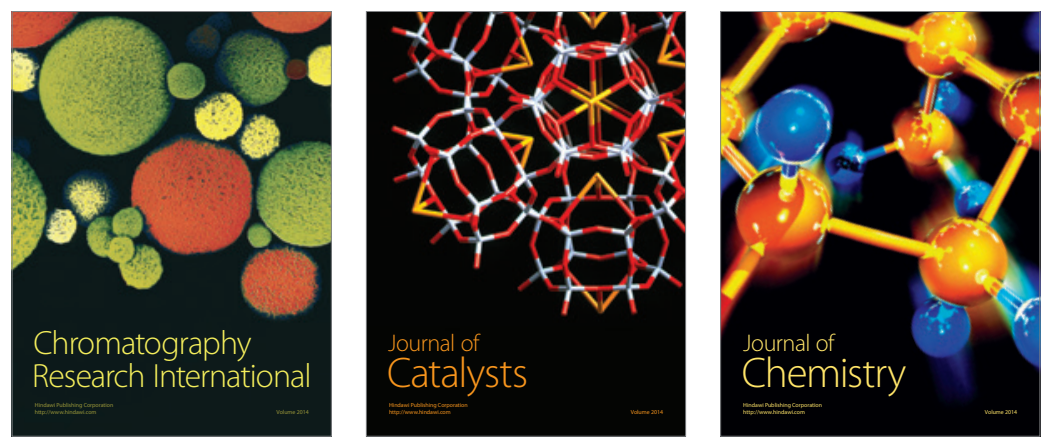
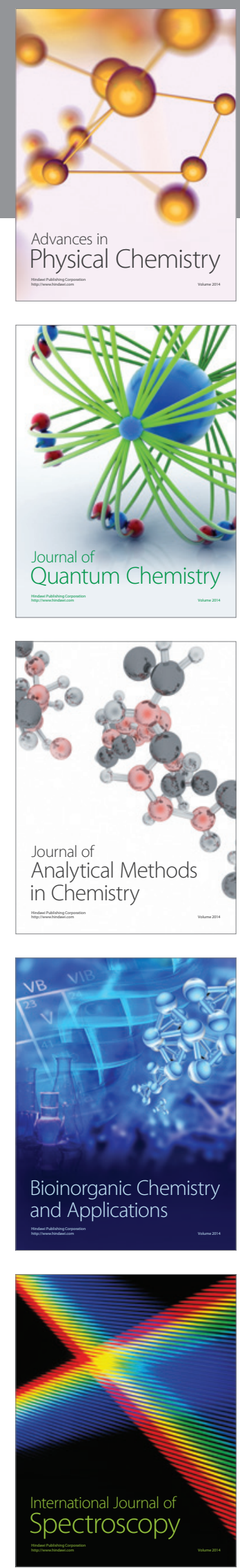